\title{
Preparation of an Amphiphilic Resin-Supported BINAP Ligand and Its Use for Rhodium-Catalyzed Asymmetric 1,4-Addition of Phenylboronic Acid in Water
}

Yusuke Otomaru, Taichi Senda, and Tamio Hayashi*

Department of Chemistry, Graduate School of Science, Kyoto University, Sakyo, Kyoto 6068502, Japan

\section{Supporting Data}

General. All anaerobic and moisture-sensitive manipulations were carried out with standard Schlenk technique under predried nitrogen or glovebox techniques under prepurified argon. NMR spectra were recorded on a JEOL JNM LA-500 spectrometer $\left(500 \mathrm{MHz}\right.$ for ${ }^{1} \mathrm{H}$, $125 \mathrm{MHz}$ for ${ }^{13} \mathrm{C}$, and $202 \mathrm{MHz}$ for ${ }^{31} \mathrm{P}$ ). Chemical shifts are reported in $\delta \mathrm{ppm}$ referenced to an internal $\mathrm{SiMe}_{4}$ standard for ${ }^{1} \mathrm{H}$ NMR, chloroform- $d(\delta 77.05)$ for ${ }^{13} \mathrm{C} \mathrm{NMR}$, and external $85 \% \mathrm{H}_{3} \mathrm{PO}_{4}$ standard for ${ }^{31} \mathrm{P}$ NMR. Optical rotations were measured on a JASCO DIP-370 polarimeter.

Materials. THF, $\mathrm{Et}_{2} \mathrm{O}$ and dioxane were distilled from sodium benzophenone-ketyl under nitrogen. $\mathrm{CH}_{2} \mathrm{Cl}_{2}$ and DMF were distilled from $\mathrm{CaH}_{2}$ under nitrogen. $\mathrm{NiCl}_{2}$ (dppe) ${ }^{1}$ and $\mathrm{Rh}(\mathrm{acac})\left(\mathrm{C}_{2} \mathrm{H}_{4}\right)_{2}{ }^{2}$ were prepared according to the reported procedures. 2-Cyclohexenone, 2cyclopentenone, 2-cycloheptenone, and trans-3-nonen-2-one were purchased and distilled before use. 5-Methyl-3-hexen-2-one, phenylboronic acid, $(S)$-2,2'-dihydroxy-1,1'-binaphthyl (BINOL), and 1-methyl-2-pyrrolidinone were purchased and used without further purification. (S)-6-Bromo-2,2'-dihydroxy-1,1'-binaphthyl (2) was prepared from $(S)$-BINOL according to the reported procedure. ${ }^{3}$

(S)-6-Bromo-2,2'-bis(trifluoromethanesulfonyloxy)-1,1'-binaphthyl (3). To a solution of (S)-6-bromo-2,2'-dihydroxy-1,1'-binaphthyl (2) (20.6 g, $56.3 \mathrm{mmol}$ ) and pyridine $(13.7 \mathrm{~mL}, 170 \mathrm{mmol})$ in $100 \mathrm{~mL}$ of $\mathrm{CH}_{2} \mathrm{Cl}_{2}$ was added trifluoromethanesulfonic anhydride $(22.7 \mathrm{~mL}, 135 \mathrm{mmol})$ at $0{ }^{\circ} \mathrm{C}$, and the mixture was stirred at room temperature for 8 h. After removal of the solvent, the residue was diluted with $200 \mathrm{~mL}$ of ethyl acetate, and it was washed with $1 \mathrm{~N} \mathrm{HCl}$, saturated $\mathrm{NaHCO}_{3}$, and brine. The organic layer was dried over $\mathrm{Na}_{2} \mathrm{SO}_{4}$ and concentrated under reduced pressure. The residue was purified by chromatography on silica gel column (benzene) to give $27.6 \mathrm{~g}$ (78\% yield) of 3 . ${ }^{1} \mathrm{H}$ NMR $\left(\mathrm{CDCl}_{3}\right): \delta 7.13(\mathrm{~d}, J=9.1 \mathrm{~Hz}, 1 \mathrm{H}), 7.20(\mathrm{~d}, J=8.6 \mathrm{~Hz}, 1 \mathrm{H}), 7.43(\mathrm{t}, J=7.7 \mathrm{~Hz}, 1 \mathrm{H}), 7.47$ $(\mathrm{dd}, J=9.1,2.0 \mathrm{~Hz}, 1 \mathrm{H}), 7.60(\mathrm{t}, J=7.6 \mathrm{~Hz}, 1 \mathrm{H}), 7.61(\mathrm{~d}, J=9.3 \mathrm{~Hz}, 1 \mathrm{H}), 7.64(\mathrm{~d}, J=$ $9.2 \mathrm{~Hz}, 1 \mathrm{H}), 8.02(\mathrm{~d}, J=8.2 \mathrm{~Hz}, 1 \mathrm{H}), 8.05(\mathrm{~d}, J=9.2 \mathrm{~Hz}, 1 \mathrm{H}), 8.16(\mathrm{~d}, J=9.2 \mathrm{~Hz}, 1 \mathrm{H})$, $8.18(\mathrm{~d}, J=1.8 \mathrm{~Hz}, 1 \mathrm{H}) .{ }^{13} \mathrm{C}\left\{{ }^{1} \mathrm{H}\right\} \operatorname{NMR}\left(\mathrm{CDCl}_{3}\right): \delta 118.15\left(\mathrm{q}, J_{\mathrm{C}-\mathrm{F}}=319 \mathrm{~Hz}\right), 118.20(\mathrm{q}$, $\left.J_{\mathrm{C}-\mathrm{F}}=319 \mathrm{~Hz}\right), 119.38,120.64,121.83,122.95,123.97,126.49,127.46,128.22,128.49$, 128.52, 130.44, 131.05, 131.49, 131.77, 132.34, 132.43, 133.03, 133.41, 145.39, 145.57. Anal. Calcd for $\mathrm{C}_{22} \mathrm{H}_{11} \mathrm{O}_{6} \mathrm{BrF}_{6} \mathrm{~S}_{2}$ : C, 41.99; H, 1.76. Found: $\mathrm{C}, 42.24 ; \mathrm{H}, 1.98$. $[\alpha]^{20} \mathrm{D}$ $+137\left(\right.$ c $\left.0.49, \mathrm{CHCl}_{3}\right)$. 
(S)-6-Cyano-2,2'-bis(trifluoromethanesulfonyloxy)-1,1'-binaphthyl (4). A solution of (S)-6-bromo-2,2'-bis(trifluoromethanesulfonyloxy)-1,1'-binaphthyl (3) (5.88 g, $9.34 \mathrm{mmol})$ and cuprous cyanide $(1.50 \mathrm{~g}, 16.8 \mathrm{mmol})$ in $20 \mathrm{~mL}$ of 1-methyl-2-pyrrolidinone was stirred at $180{ }^{\circ} \mathrm{C}$ for $4 \mathrm{~h}$. After cooled to about $100{ }^{\circ} \mathrm{C}$, the reaction mixture was poured into $100 \mathrm{~mL}$ of $14 \%$ aqueous $\mathrm{NH}_{3}$, and it was extracted with benzene. The organic layer was washed with $5 \%$ aqueous $\mathrm{NH}_{3}(50 \mathrm{~mL}$ x 4$)$ and brine $(50 \mathrm{~mL} \times 2)$, before it was dried over $\mathrm{Na}_{2} \mathrm{SO}_{4}$ and concentrated under reduced pressure. The residue was purified by chromatography on silica gel column (benzene) to give $3.73 \mathrm{~g}$ (73\% yield) of $4 .{ }^{1} \mathrm{H}$ NMR $\left(\mathrm{CDCl}_{3}\right): \delta 7.16(\mathrm{~d}, J=8.6 \mathrm{~Hz}, 1 \mathrm{H}), 7.37(\mathrm{~d}, J=8.9 \mathrm{~Hz}, 1 \mathrm{H}), 7.45(\mathrm{t}, J=7.7 \mathrm{~Hz}, 1 \mathrm{H}), 7.55$ $(\mathrm{dd}, J=8.9,1.5 \mathrm{~Hz}, 1 \mathrm{H}), 7.62(\mathrm{t}, J=7.2 \mathrm{~Hz}, 1 \mathrm{H}), 7.62(\mathrm{~d}, J=9.2 \mathrm{~Hz}, 1 \mathrm{H}), 7.77(\mathrm{~d}, J=$ $9.2 \mathrm{~Hz}, 1 \mathrm{H}), 8.04(\mathrm{~d}, J=8.4 \mathrm{~Hz}, 1 \mathrm{H}), 8.19(\mathrm{~d}, J=9.0 \mathrm{~Hz}, 1 \mathrm{H}), 8.23(\mathrm{~d}, J=9.2 \mathrm{~Hz}, 1 \mathrm{H})$, $8.42(\mathrm{~s}, 1 \mathrm{H}) .{ }^{13} \mathrm{C}\left\{{ }^{1} \mathrm{H}\right\} \mathrm{NMR}\left(\mathrm{CDCl}_{3}\right): \delta 111.36,118.10\left(\mathrm{q}, J_{\mathrm{C}-\mathrm{F}}=319 \mathrm{~Hz}\right), 118.15\left(\mathrm{q}, J_{\mathrm{C}-\mathrm{F}}\right.$ $=319 \mathrm{~Hz}), 118.25,119.42,121.50,122.33$, 124.36, 126.17, 127.63, 128.29, 128.46, $128.65,131.31,132.45,132.51,132.68,132.84,134.30,134.78,145.36,147.39$. Anal. Calcd for $\mathrm{C}_{23} \mathrm{H}_{11} \mathrm{NO}_{6} \mathrm{~F}_{6} \mathrm{~S}_{2}: \mathrm{C}, 48.01 ; \mathrm{H}, 1.93 ; \mathrm{N}, 2.43$. Found: C, 48.21; H, 2.20; N, 2.24 . $[\alpha]^{20} \mathrm{D}+165\left(c 0.98, \mathrm{CHCl}_{3}\right)$.

(S)-6-Cyano-2,2' - bis(diphenylphosphino)-1,1'-binaphthyl (5). To a solution of $\mathrm{NiCl}_{2}$ (dppe) (317 mg, $0.60 \mathrm{mmol}$ ) in DMF (12 mL) was added diphenylphosphine $(610 \mu \mathrm{L}, 3.5 \mathrm{mmol})$ at room temperature, and then the resulting solution was heated at $100{ }^{\circ} \mathrm{C}$. After stirring at $100{ }^{\circ} \mathrm{C}$ for $30 \mathrm{~min}$, to the mixture was added a solution of $(S)$-6-cyano-2,2'bis(trifluoromethanesulfonyloxy)-1,1'-binaphthyl (4) (3.45 g, $6.0 \mathrm{mmol})$ and DABCO (2.69 g, $24 \mathrm{mmol})$ in DMF $(18 \mathrm{~mL})$. The reaction mixture was kept at $100{ }^{\circ} \mathrm{C}$, and three additional protions of diphenylphosphine $(610 \mu \mathrm{L} \times 3)$ were added at 1,3 , and $7 \mathrm{~h}$ later. The reaction mixture was stirred at $100{ }^{\circ} \mathrm{C}$ for 3 days, and then added to degassed methanol under nitrogen. The precipitate was filtered and washed with methanol. The desired product was purified by recrystallization (DMF) to give $2.82 \mathrm{~g}$ (73\% yield) of 5. ${ }^{1} \mathrm{H}$ NMR $\left(\mathrm{CDCl}_{3}\right): \delta 6.65(\mathrm{~d}, J=8.6$ $\mathrm{Hz}, 1 \mathrm{H}), 6.74(\mathrm{~d}, J=8.8 \mathrm{~Hz}, 1 \mathrm{H}), 6.85-6.90(\mathrm{~m}, 2 \mathrm{H}), 6.96-7.03(\mathrm{~m}, 4 \mathrm{H}), 7.06-7.12$ (m, $6 \mathrm{H}), 7.15-7.25(\mathrm{~m}, 10 \mathrm{H}), 7.33(\mathrm{t}, J=8.1 \mathrm{~Hz}, 1 \mathrm{H}), 7.42(\mathrm{dd}, J=8.6,2.6 \mathrm{~Hz}, 1 \mathrm{H}), 7.57(\mathrm{dd}$, $J=8.5,2.3 \mathrm{~Hz}, 1 \mathrm{H}), 7.82(\mathrm{~d}, J=8.2 \mathrm{~Hz}, 1 \mathrm{H}), 7.90(\mathrm{~d}, J=8.6 \mathrm{~Hz}, 2 \mathrm{H}), 8.17(\mathrm{~s}, 1 \mathrm{H}) .{ }^{31} \mathrm{P}$ $\operatorname{NMR}\left(\mathrm{CDCl}_{3}\right): \delta-13.8\left(\mathrm{~d}, J_{\mathrm{p}-\mathrm{p}}=17.4 \mathrm{~Hz}, 1 \mathrm{P}\right),-13.9\left(\mathrm{~d}, J_{\mathrm{p}-\mathrm{p}}=17.4 \mathrm{~Hz}, 1 \mathrm{P}\right)$. Anal.Calcd for $\mathrm{C}_{45} \mathrm{H}_{31} \mathrm{NP}_{2}$ : C, 83.45; H, 4.82; N, 2.16; P, 9.56. Found: C, 83.19; H, 4.82; N, 2.09; P, 9.31. $[\alpha]^{20} \mathrm{D}-130\left(c 0.925, \mathrm{CHCl}_{3}\right)$.

\section{(S)-6-Hydroxycarbonyl-2,2' -bis(diphenylphosphino)-1,1'-binaphthyl}

(6). A mixture of (S)-6-cyano-2,2'-bis(diphenylphosphino)-1,1'-binaphthyl (5) (259 mg, $0.40 \mathrm{mmol})$ and $\mathrm{KOH}(280 \mathrm{mg}, 5.0 \mathrm{mmol})$ in dioxane $(4 \mathrm{~mL}), \mathrm{MeOH}(4 \mathrm{~mL})$, and $\mathrm{H}_{2} \mathrm{O}(1.5$ $\mathrm{mL}$ ) was refluxed for $24 \mathrm{~h}$. The reaction mixture was acidified with $2 \mathrm{~N} \mathrm{HCl}$, and then extracted with ethyl acetate. The organic layer was dried over $\mathrm{MgSO}_{4}$ and concentrated under reduced pressure. The residue was purified by recrystallization (2-PrOH) to give $226 \mathrm{mg}$ ( $85 \%$ yield) of 6. ${ }^{1} \mathrm{H} \mathrm{NMR}\left(\mathrm{CDCl}_{3}\right): \delta 6.71(\mathrm{~d}, J=8.5 \mathrm{~Hz}, 1 \mathrm{H}), 6.81(\mathrm{~d}, J=9.0 \mathrm{~Hz}, 1 \mathrm{H}), 6.87(\mathrm{t}$, 
$J=7.6 \mathrm{~Hz}, 1 \mathrm{H}), 6.98-7.22(\mathrm{~m}, 20 \mathrm{H}), 7.33(\mathrm{t}, J=7.5 \mathrm{~Hz}, 1 \mathrm{H}), 7.45(\mathrm{t}, J=8.9 \mathrm{~Hz}, 2 \mathrm{H})$, $7.52(\mathrm{~d}, J=8.5 \mathrm{~Hz}, 1 \mathrm{H}), 7.83(\mathrm{~d}, J=8.1 \mathrm{~Hz}, 1 \mathrm{H}), 7.90(\mathrm{~d}, J=8.6 \mathrm{~Hz}, 1 \mathrm{H}), 8.00(\mathrm{~d}, J=$ $8.7 \mathrm{~Hz}, 1 \mathrm{H}), 8.65(\mathrm{~s}, 1 \mathrm{H}), 11.47$ (br s, $1 \mathrm{H}) .{ }^{31} \mathrm{P} \mathrm{NMR}\left(\mathrm{CDCl}_{3}\right): \delta-14.1\left(\mathrm{~d}, J_{\mathrm{p}-\mathrm{p}}=15.6 \mathrm{~Hz}\right.$, $1 \mathrm{P}),-14.2\left(\mathrm{~d}, J_{\mathrm{p}-\mathrm{p}}=15.6 \mathrm{~Hz}, 1 \mathrm{P}\right)$. Anal.Calcd for $\mathrm{C}_{45} \mathrm{H}_{32} \mathrm{O}_{2} \mathrm{P}_{2}: \mathrm{C}, 81.07 ; \mathrm{H}, 4.84 ; \mathrm{P}, 9.29$. Found: C, 80.84; H, 5.01; P, 9.37. [ $\alpha]^{20} \mathrm{D}-149\left(c 0.92, \mathrm{CHCl}_{3}\right)$.

PS-PEG resin-supported $(\boldsymbol{S})$-binap (1). TentaGel ${ }^{\circledR}$ S-NH 2 beads $(2.0 \mathrm{~g}, 0.26$ $\mathrm{mmol} \mathrm{N} / \mathrm{g})$ were washed with acetonitrile $(20 \mathrm{~mL}$ x 6$)$ and chloroform $(20 \mathrm{~mL}$ x 5) prior to use. A suspension of the washed TentaGel ${ }^{\circledR}$ S-NH 2 beads, $(S)$-6-Hydroxycarbonyl-2,2'bis(diphenylphosphino)-1,1'-binaphthyl (6) (507 mg, $0.76 \mathrm{mmol}$ ), EDCI HCl (230 mg, 1.2 $\mathrm{mmol}$ ), and HOBt $(211 \mathrm{mg}, 1.56 \mathrm{mmol})$ in DMF (40 mL) was stirred at room temperature for 4 h. The raction mixture was filtered and the resin was washed with DMF (20 mL x 5) and dichloromethane (20 mL x 8). The resin was dried under reduced pressure to give $1.9 \mathrm{~g}$ of $\mathbf{1}$ (0.168 mmol BINAP/g). ${ }^{31} \mathrm{P}$ NMR (THF): $\delta-14.5$ (br s). Found: C, 66.77; H, 8.06; N, $0.33 ; \mathrm{P}, 1.04$.

\section{Reaction of $\mathrm{Rh}(\operatorname{acac})\left(\mathrm{C}_{2} \mathrm{H}_{4}\right)_{2}$ with PS-PEG resin-supported $(S)$-binap} (1). In an NMR sample tube, $\mathrm{Rh}(\mathrm{acac})\left(\mathrm{C}_{2} \mathrm{H}_{4}\right)_{2}(2.6 \mathrm{mg}, 10 \mu \mathrm{mol} \mathrm{Rh})$ and PS-PEG resinsupported binap (1) $(59.5 \mathrm{mg}, 10 \mu \mathrm{mol}$ binap) were charged and THF $(0.5 \mathrm{~mL})$ was added at room temperature. Within $3 \mathrm{~min}, \mathrm{Rh}(\mathrm{acac})\left(\mathrm{C}_{2} \mathrm{H}_{4}\right)_{2}$ was converted into $\mathrm{Rh}(\mathrm{acac})(\mathrm{PS}-\mathrm{PEG}-(\mathrm{S})$ binap-1). ${ }^{31} \mathrm{P}$ NMR (THF): $\delta 55.0\left(\mathrm{dd}, J_{\mathrm{Rh}-\mathrm{P}}=194 \mathrm{~Hz}, J_{\mathrm{P}-\mathrm{P}}=64 \mathrm{~Hz}, 1 \mathrm{P}\right), 55.3\left(\mathrm{dd}, J_{\mathrm{Rh}-\mathrm{P}}=\right.$ $\left.193 \mathrm{~Hz}, J_{\mathrm{P}-\mathrm{P}}=64 \mathrm{~Hz}, 1 \mathrm{P}\right)$.

Rhodium-catalyzed asymmetric 1,4-addition of phenylboronic acid to $\boldsymbol{\alpha}, \boldsymbol{\beta}$-unsaturated ketones. The reaction conditions and results are summarized in Table 1. A typical procedure is shown below: A mixture of $\mathrm{Rh}(\mathrm{acac})\left(\mathrm{C}_{2} \mathrm{H}_{4}\right)_{2}(1.5 \mathrm{mg}, 6 \mu \mathrm{mol})$, phenylboronic acid (122 mg, $1.0 \mathrm{mmol})$, and 1 (53.6 mg, $9 \mu \mathrm{mol}$ BINAP) in $0.5 \mathrm{~mL}$ of THF was stirred at room temperature for $3 \mathrm{~min}$. After evaporation of the solvent, 2-cyclopentenone (16.4 mg, $0.20 \mathrm{mmol})$ and $\mathrm{H}_{2} \mathrm{O}(1.0 \mathrm{~mL})$ were added and the mixture was stirred at $100{ }^{\circ} \mathrm{C}$ for $3 \mathrm{~h}$. The reaction mixture was filtered and the resin was washed with $\mathrm{Et}_{2} \mathrm{O}$. The filtrate was washed with saturated $\mathrm{NaHCO}_{3}$ and brine. The organic layer was dried over $\mathrm{MgSO}_{4}$ and concentrated under reduced pressure. The residue was purified by preparative TLC (silica gel, hexane/ethyl acetate $=3 / 1$ ) to give $30.4 \mathrm{mg}$ ( $95 \%$ yield) of 3-phenylcyclopentanone.

For the recycling of the catalyst, see footnote $d$ of Table 1.

\section{References}

(1) Booth, G.; Chatt, J. J. Chem. Soc. 1965, 3239.

(2) Cramer, R. Inorg. Synth. 1974, 15, 16.

(3) (a) Cai, D.; Larsen, R. D.; Reider, P. J. Tetrahedron Lett. 2002, 43, 4055. (b) Hocke,

H.; Uozumi, Y. Tetrahedron, 2003, 59, 619. 